\title{
Ontogenetic changes in the rate of ingestion and estimates of food consumption in fourth and fifth instar Helicoverpa armigera caterpillars
}

\author{
Lindsay Barton Browne ${ }^{\mathrm{a}}$, David Raubenheimer ${ }^{\mathrm{b},}$ * \\ ${ }^{a}$ CSIRO Entomology, GPO Box 1700, Canberra, ACT, 2601, Australia ${ }^{\mathrm{b}}$ Department of Zoology, University of Oxford, South Parks \\ Road, Oxford OXI 3PS, UK
}

Received 21 March 2002; received in revised form 15 October 2002; accepted 16 October 2002

\begin{abstract}
We present the second in a series of experiments investigating the behavioural mechanisms used by Helicoverpa armigera caterpillars to fund the increased nutrient requirements associated with growth and development. In the work reported here, we measured ontogenetic changes in the rate of ingestion (amount of an artificial food ingested per unit time when the insect is actually feeding) in fourth and fifth (penultimate and ultimate) instar caterpillars. These data are used together with those obtained in a previous study on ontogenetic changes in the proportion of time spent feeding to estimate the total amount of food ingested over three 33.3\% temporal segments of the period from ecdysis to the cessation of feeding in the two stadia. Overall, the rate of ingestion in the fifth stadium was about three times that in the fourth. Rate of ingestion was constant over the fourth stadium but increased over the course of the fifth. Total consumption in the fifth stadium was about 3.5 times greater than in the fourth, mainly due to the greater rate of ingestion. In the fourth stadium, consumption in the third segment was greater than in either of the first two segments because the time spent feeding was greater. In the fifth stadium, consumption in the second segment was greater than in the first because of an increase in time spent feeding. In contrast, the greater intake in the third segment as compared with the second was due to an increase in the rate of ingestion. Our results demonstrated that the larvae, through increasing the rate of ingestion, were able to satisfy their increasing nutritional requirements without there being, necessarily, a commensurate increase in the time spent feeding. (C2002 Elsevier Science Ltd. All rights reserved.
\end{abstract}

Keywords: Ontogenetic changes; Rate of ingestion; Food consumption; Caterpillars; Helicoverpa armigera

\section{Introduction}

The nutritional requirements of an insect change throughout development and such changes are typically reflected in changes in food consumption and feeding behaviour (Barton Browne, 1995). In a larval insect, the nutritional requirements over different developmental periods are positively correlated with growth over the period, since growth is directly funded by nutrients. It is likely, also, that nutritional requirements would be positively correlated with the mass of the insect. This

\footnotetext{
* Corresponding author at present address: Wissenschaftskolleg

Wallotstrasse 19, 14193 Berlin, Germany. E-mail address: david.raubenheimer@zoo.ox.ac.uk (D. Raubenheimer).

is because the nutritional requirements for maintenance could be expected to be correlated with tissue mass (Schroeder, 1981; Phillipson, 1981). The factors determining nutrient availability for growth and maintenance over a given period of development are the amount and type of food consumed and the efficiency with which it is utilized. The amount of food consumed by an insect over a given period is, in turn, determined by two factors; the total time spent feeding and the mean rate at which food is
} 
ingested while the insect is actually feeding.

What this all amounts to is the fact that a number of interacting factors determines the amounts of nutrient provided for use by the tissues of an animal, and changes in various combinations of these could be used to meet the increased nutrient requirements due to growth. Pre-

0022-1910/03/\$ -see front matter @2002 Elsevier Science Ltd.

All rights reserved. doi:10.1016/S0022-1910(02)00247-0

L. Barton Browne, D. Raubenheimer / Journal of Insect Physiology 49 (2003) 63-71

cisely which combination of parameters changes with development in a given instance is, however, probably not arbitrary, but is likely to have important ecological and nutritional implications. For example, ecological studies have shown that the risk of predation to caterpillars is greatly increased when feeding (Bernays, 1991, 1997), giving rise to the expectation that for these insects it might be advantageous to maximize the rate of ingestion, so minimizing hazardous exposure. On the other hand, some herbivorous insects rely on mandibular crushing of plant tissue for the extraction of nutrients (Bernays, 1991). When this is the case, insects are likely to be more constrained in the extent to which they can profitably increase the rate of ingestion, and so might more readily meet their growing nutrient requirements by increasing the time spent feeding.

We have previously (Raubenheimer and Barton Browne, 2000) examined ontogenetic changes in temporal patterns of feeding over the fourth and fifth (penultimate and ultimate) stadia of Helicoverpa armigera. The data obtained in that study allowed us to estimate one of the major factors determining the amount eaten, the time spent feeding over defined developmental stages. In the present paper we extend this work, presenting data on the ontogenetic changes over the same two stadia of H. armigera in the second factor determining the amount eaten, the rate of ingestion while feeding. By combining the data on ingestion rates and time spent feeding we were, furthermore, able to estimate the changes in food consumption across defined temporal segments in each stadium. The data showed that ingestion rate is a major factor underlying ontogenetic changes in food intake by H. armigera, thus confirming expectations derived from ecological studies of predation that caterpillars should minimize the amount of time spent feeding (Bernays, 1991, 1997). However, there were also increases across some developmental stages within stadia in the time spent feeding, suggesting that the story is not simple but possibly involves age or size-specific selection factors or constraints.

\section{Materials and methods}

\subsection{Insects}

Larvae of $H$. armigera were from the same source as those used in our study of developmental changes in the temporal patterns of feeding (Raubenheimer and Barton Browne, 2000). They were obtained from a long established culture maintained in the laboratories of the Division of Plant Protection, Queensland Department of Primary Industries (QDPI), Indooroopilly, Qld. In this culture, larvae were routinely reared on a navy bean-based diet modified from that of Teakle and Jensen (1985) by increasing the amount of all dry ingredients by a factor of 1.25 (not 0.25 as erroneously stated by Raubenheimer and Barton Browne (2000)).

\subsection{Rates of ingestion}

\subsubsection{Estimation of rates of ingestion}

Larvae, obtained from QDPI in the second stadium, were allowed to remain feeding in groups until the afternoon of the day before they were expected to undergo ecdysis into the fourth instar. Forty such larvae were set up individually in chambers consisting of a $50 \mathrm{~mm}$ square celluloid base and an inverted, lidless, cylindrical clear plastic pot $40 \mathrm{~mm}$ in diameter and $65 \mathrm{~mm}$ high (referred to as 'type 
1 ' chambers in the description that follows). The individual larvae were placed on a block of the beanbased diet $(30 \times 20 \times 20 \mathrm{~mm})$ which had already been placed on the celluloid base under the inverted pot. Frass was removed from the chambers and the larvae were given fresh diet at 0700 and $1900 \mathrm{~h}$ each day. They were weighed at the latter time and these data were used to establish fresh mass trajectories. Otherwise, the larvae remained in these chambers continuously except for the time they spent in 'type 2' chambers (see below) for assessment of rate of ingestion.

At the beginning of the observation period each day, a $10 \mathrm{~mm}$ cube of diet on a $15 \mathrm{~mm}$ square 'tray' of celluloid was placed into each of several chambers (type 2 chambers) which differed from the type 1 chamber in that the base was a $250 \mathrm{~mm}$ diameter glass Petri dish instead of a celluloid sheet. The array of larvae in their type 1 chambers was scanned every few minutes throughout the daily observation period (circa 0700 $1900 \mathrm{~h}$ ) and any larvae feeding were noted. When a larva was observed to cease feeding, the diet on its celluloid tray was removed from one of the type 2 chambers and the larva placed in it. The assembly consisting of Petri dish, inverted pot and larva was then weighed to an accuracy of $0.1 \mathrm{mg}$. Following the weighing, a fresh block of medium on a celluloid tray was introduced into the chamber. Larvae set up in the type 2 chambers were scanned continuously, there being no more than four of these chambers in use at any one time. When a larva started to feed on the diet, the time was recorded to the nearest second. The feeding larva was then watched continuously. In most instances a larva, having begun feeding, ingested food continuously for $\checkmark 1 \mathrm{~min}$. The time at which this period of continuous ingestion ended was recorded. Henceforth, we refer to the period of continuous ingestion as the feed and its duration as feed duration. As soon as the larva was observed to cease feeding, it was lifted off the diet and the diet on its tray removed from the chamber. The pot was immediately replaced over the larva and the assembly consisting of Petri dish, plastic pot, larva and any faecal pellets produced during the larva's period of occupancy of the type 2 chamber weighed. The difference between the post

L. Barton Browne, D. Raubenheimer / Journal of Insect Physiology 49 (2003) 63-71

feeding and pre-feeding weights of the assembly was taken as the amount ingested during the feed. The rate of ingestion in $\mathrm{mg} / \mathrm{min}$ was calculated by dividing this figure by feed duration. Immediately following this post-feeding weighing, the larva was removed from the chamber and was itself weighed, to give an additional point on its mass trajectory, prior to its being replaced in its original type 1 chamber. A fresh cube of diet was then placed in the just vacated type 2 chamber.

Disposable gloves were worn whenever any part of the assembly was being handled to ensure that no moisture or other material was transferred to any of the glass or plastic surfaces. Diet was kept in the type 2 chambers at all times (other than during the pre-and post-ingestion weighings) to minimize variation in the amount of water adsorbed on their internal surfaces. Observations were made for at least $12 \mathrm{~h}$ on each of 5 days following that on which the 40 larvae were originally set up in their type 1 chambers. These 5 days encompassed the feeding periods of larvae in their fourth and fifth stadia. The number of occasions on which estimates of ingestion rate were obtained varied among larvae, ranging from more than one in each stadium to a single estimate over the course of the two stadia.

All observations were carried out at $27 \pm 1^{\circ} \mathrm{C}$ under constant artificial illumination.

\subsubsection{Binning of data into stadium segments}

Data on rates of ingestion for each of the two stadia were assigned to three bins according to elapsed time since the estimated time of ecdysis, expressed as a percentage of the whole period from ecdysis to the cessation of feeding within the stadium. This enabled us to use similar analyses for rates of ingestion as we used to investigate ontogenetic changes in the temporal patterns of feeding (Raubenheimer and Barton Browne, 2000), and thus directly to interrelate the two data sets.

In our previous study on temporal patterns of feeding we binned the data obtained for each individual larva, in each stadium, according to whether it applied to the first 33.3\% (0-33.3\%) of the total duration of the period from ecdysis to the cessation of feeding, to between 33.4 and $66.6 \%$ or to between 66.7 and $100 \%$ of this period. In the present study of rate of ingestion, because we did not observe the insects continuously as in our previous study, we rarely knew when an individual insect 
took its last meal within a stadium. Thus, in assigning the data on rate of ingestion to bins, we used the median durations of the interval from ecdysis to the cessation feeding obtained for the two stadia in our earlier study, viz, $37.8 \mathrm{~h}$ for the fourth stadium and $56 \mathrm{~h}$ for the fifth stadium. Thus, in the fourth stadium, the period from 0 to $12.6 \mathrm{~h}$ after the estimated time of ecdysis into the fourth stadium was designated segment 1 , from 12.7 to $25.3 \mathrm{~h}$ after ecdysis segment 2 , and any time after ecdysis $25.4 \mathrm{~h}$ segment 3. In the fifth stadium, segment 1 was considered to be from 0 to $18.7 \mathrm{~h}$, segment 2 from 18.8 to 37.3 and segment $3 \circlearrowleft 37.8 \mathrm{~h}$ after ecdysis into the fifth stadium.

\subsubsection{Analysis of within-stadium comparisons}

Ontogenetic changes in rate of ingestion within each of the two stadia were examined by carrying out nonparametric Spearman's correlation analyses with the independent variable being hours since the estimated time of the previous ecdysis.

In addition to these correlation analyses, we tested for changes within the stadia by comparing rates in segment 3 with those in segment 1 . We carried out Mann-Whit-ney tests on rates in the two segments and paired Wilcoxon tests when we had data for the same insect in the two segments. In each case, if an insect provided more than one data point in a particular segment, one of these was randomly selected and included in the analysis, while the other was excluded. It should be noted that numbers of paired observations were very low in some cases, giving low statistical power, so instances in which there were statistically significant outcomes are, in this respect, more informative than

non-significant

comparisons.

\subsubsection{Analysis of between-stadium comparisons}

Mann-Whitney tests (two-tailed) were performed between like segments in the two stadia, i.e. rates in fourth stadium segment 1 were compared with fifth stadium, segment 1 etc. In addition, to investigate any overlap between stadia, the rates of ingestion in segment 3 of the fourth stadium were compared with those in segment 1 of the fifth stadium.

\subsection{Estimation of consumption in segments within stadia}

To obtain estimates of consumption, we multiplied estimates of median time spent feeding in each of the three equal segments of the fourth and fifth stadia, derived from the data obtained in our previous study of ontogenetic changes in temporal patterns of feeding (Raubenheimer and Barton Browne, 2000), by the median rates of ingestion in the same six temporal segments obtained in the present study. Estimates of time spent feeding (in minutes) in each segment were obtained by multiplying the median proportion of time spent feeding in that segment by the median length in minutes of the segment (Raubenheimer and Barton Browne, 2000). The median length of each segment in the fourth stadium was 756 (range, 600-900) min and in the fifth stadium 1120 (range, 980-1240) min. Since estimates of consumption were derived from data from two separate, even if closely parallel studies, we made no attempt to analyse statistically the calculated estimates of consumption shown in Fig. 1.

L. Barton Browne, D. Raubenheimer / Journal of Insect Physiology 49 (2003) 63-71 


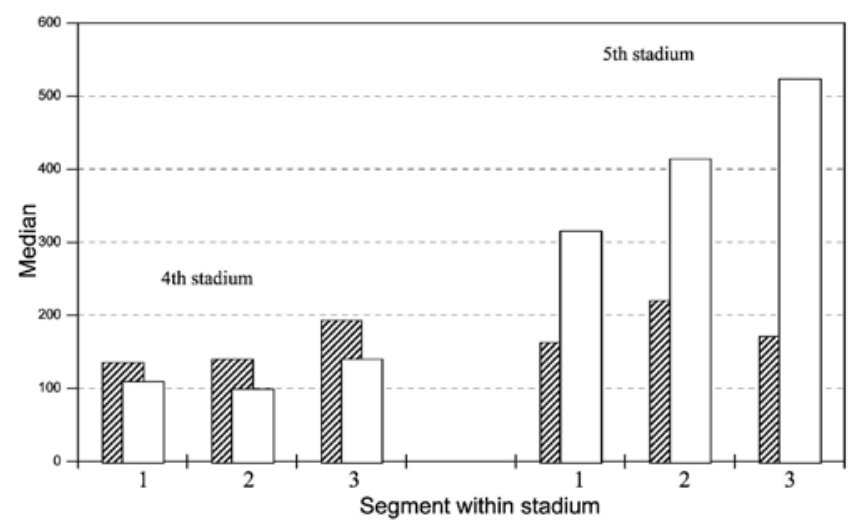

Fig. 1. Median estimates of consumption in milligrams (open bars) and of time in minutes spent feeding (hatched bars) across successive $33.3 \%$ segments of the feeding periods of the fourth and fifth stadia of $H$. armigera caterpillars.

\subsection{Fresh mass trajectories}

Fresh mass trajectories over the two stadia were constructed by plotting fresh mass against estimated time since ecdysis into the stadium. Readings of fresh mass were obtained from (1) weighings carried out in association with the estimation of rate of ingestion (see above) and (2) routine weighings made at $1900 \mathrm{~h}$ on each day of the observation period when the larvae were supplied with fresh medium. There was no suggestion of statistical differences between trajectories obtained from the two categories of weighings ( $p=0.662$ for fourth instars, and $p=0.601$ for fifth instars), so they were combined for the final analysis. Estimates of the timing of ecdyses that were not actually observed were obtained in the manner described by Raubenheimer and Barton Browne (2000).

Fresh mass increased only over the feeding period of each stadium. Because of this, readings of mass were used in the analysis of the trajectories only if they were obtained in association with an estimation of rate of ingestion (in which case the insect was, by definition, within the feeding period of the stadium) or, in the case of readings of mass obtained in routine weighings at $1700 \mathrm{~h}$, if the estimated time after ecdysis did not exceed the minimum duration seen in our previous study of temporal patterns of feeding (Raubenheimer and Barton Browne, 2000).

We refer to trajectories obtained using the sequential mass determinations as mass trajectories rather than growth trajectories. This is in recognition of the fact that gut contents account for a very significant proportion of whole insect fresh mass in caterpillars [e.g. ca 40\% in fifth instar Manduca sexta (Reynolds et al., 1985)]. Thus, whole insect mass trajectories do not equate to tissue growth trajectories.

Separate analyses were performed for linearity in the mass trajectories of fourth and fifth instar larvae using stepwise linear regression including the linear, quadratic and cubic exponents of insect age. This analysis was performed separately using both absolute time since ecdysis and percentage time into the stadium as independent variables. Since there were no qualitative differences in the outcomes of these analyses, we present only the data for absolute time.

Comparison of mass trajectories were performed using analysis of covariance on insect mass including the linear, quadratic and cubic exponents of age as covariates and stadium as factor. In this model, differences in rates of mass increase (i.e. slope differences in the relationship between age and mass) would be detected as significant covariate-stadium interactions. To comply with the assumption of independence of data points, in cases where individuals were measured more than once only a single randomly selected measure was included in the analysis. 


\section{Results}

\subsection{Rate of ingestion}

Table 1 shows, for three equal segments of the fourth and fifth stadia, summary statistics (medians and ranges) for amounts eaten in feeds, feed durations, and ingestion rates, as well as Mann-Whitney probabilities for between-stadium comparisons. Statistical tests for within stadium changes are presented in Table 2.

\subsubsection{Within-stadium changes}

The data show that rate of ingestion in the fourth stadium was independent of time since ecdysis, whereas in the fifth stadium it increased significantly over the course of the stadium. These conclusions are supported by the three kinds of analyses presented in Table 2. Ingestion rate correlated significantly with insect age in the fifth but not the fourth stadia. Both Mann-Whitney tests and paired Wilcoxon tests showed that in the fifth stadium ingestion rate was significantly higher in segment 3 than in segment 1, but there was no such difference in the fourth stadium.

The data suggest that the mechanism for developmental changes in ingestion rate within the fifth stadium was primarily alteration in the time taken to eat a similar amount of food per feed. This can be seen in the fact that the pattern of statistical significance in feed duration, but not amount ingested, closely paralleled that for ingestion rate (Table 2).

\subsubsection{Between-stadium changes}

Comparison of rates of ingestion in equivalent time segments of the two stadia (e.g. segment 1 of the fourth stadium versus segment 1 of the fifth stadium) showed that, in all segments, the rate of ingestion in fifth stadium was significantly and appreciably (at least twofold)

Table 1Medians and ranges (in italics) for consumption, feed duration (see text) and ingestion rates of $H$. armigera caterpillars fed artifi cial food during the fi rst, second and third 33\% segments of the fourth and fi fth larval stadia Stadium \% period within stadium

$0-33.3$

3
3
4
-
6
6
6

Mass eaten Feed duration Ingestion

rate Mass eaten (mg) (min)

(mg/min) (mg)

$66.7-100$

Feed duration Ingestion rate Mass eaten Feed duration Ingestion rate (min) (mg/min) (mg) (min) (mg/min)

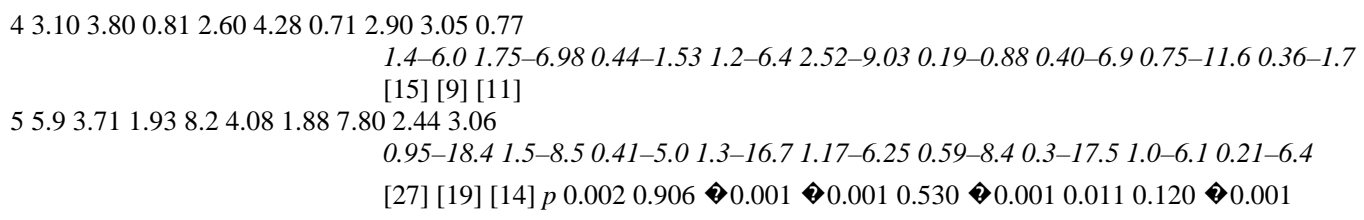


The number of observations is presented in square brackets in the fi rst column for each time segment. $p$ refers to two-tailed MannWhitney probability.

greater than that in the fourth (Table 1). An additional comparison showed that the rate in segment 1 of the fifth stadium was significantly greater in that in segment 3 of the fourth stadium. The rate of ingestion in the fifth stadium was greater than the fourth stadium because greater amounts were eaten in feeds of similar duration.

\subsection{Estimates of consumption across segments of the fourth and fifth stadia}

Fig. 1 shows estimates of consumption across the three equal temporal segments of the two stadia, as obtained by multiplying the median time spent feeding in each segment (derived from data from Raubenheimer and Barton Browne, 2000) by the median rate of ingestion in the same segments (measured in the present study). Equivalent calculations using means provided very similar estimates, but we decided to present median data to maintain continuity.

\subsubsection{Within-stadium changes}

In the fourth stadium, estimated consumption was similar in the first two segments but was substantially (ca 1.4-fold) greater in segment 3. In the fifth stadium, estimated consumption increased progressively over the three segments, that in segment 3 being about 1.7 times that in the segment 1 .

\subsubsection{Between-stadium changes}

Overall, estimated consumption in the fifth stadium is ca 3.5 times greater than that in the fourth. Differences were apparent in all comparisons of corresponding segments of the two stadia, with the factor by which estimated consumption in the fifth stadium exceeded that in the fourth being ca 2.8 in segment $1, \quad 4.1$ in segment 2 and 3.7 in segment 3.

\subsection{Fresh mass trajectories}

Fresh mass trajectories of fourth and fifth instar larvae are linear. In both cases only the linear term was retained in the stepwise linear regression (using stepping method entry criterion of $p=0.05$ ). Regression statistics for the fourth and fifth stadium, respectively, were $F(1,24)=147.2, p-0.0001$ and $F(1,33)=556.2, p-0.0001$. Inhomogeneity of variances prevented a direct comparison of the slopes in a single ANCOVA model, so we used separate linear regressions to obtain parameter estimates \pm standard error for rates of mass increase in the two stadia. The best-fit equation for the fourth and fifth stadia, respectively, were: size $(\mathrm{mg})=3.8 \pm 0.316$, age $(\mathrm{h})+22.5 \pm 6.15$ and size (mg) $=9.5 \pm 0.4$, age $(\mathrm{h})+153 \pm 10.7$.

According to estimates based on these equations, larval mass increased by $144 \mathrm{mg}$ (from ca 22 to ca $166 \mathrm{mg}$ ) over the $37.8 \mathrm{~h}$ median feeding period in the fourth stadium and by $532 \mathrm{mg}$ (from 153 to $685 \mathrm{mg}$ ) over 56

L. Barton Browne, D. Raubenheimer / Journal of Insect Physiology 49 (2003) 63-71

L. Barton Browne, D. Raubenheimer / Journal of Insect Physiology 49 (2003) 63-71

Table 2 Developmental changes in amount eaten within a feed (see text), feed duration and ingestion rate for fourth and fifth stadium H. armigera caterpillars 


\begin{tabular}{|c|c|c|c|c|c|c|c|c|}
\hline $\begin{array}{l}\text { Coefficient } \\
\text { (c) }\end{array}$ & $n$ & $p(c=0)$ & 3) $p$ (se & $n(1 \diamond 3)$ & $n(3 \diamond 1)$ & \multicolumn{2}{|c|}{$\begin{array}{l}p(\text { seg. 1=seg. } \\
3)\end{array}$} & \\
\hline \multirow{2}{*}{$\begin{array}{l}\text { Fourth } \\
\text { stadium }\end{array}$} & Mass eaten & -0.074 & 35 & 0.673 & 0.716 & 3 & & 0.686 \\
\hline & Feed duration & -0.205 & 35 & 0.238 & 0.169 & 2 & & 0.686 \\
\hline \multirow[t]{3}{*}{$\begin{array}{l}\text { Fifth } \\
\text { stadium }\end{array}$} & $\begin{array}{l}\text { Ingestion rate } \\
\text { Mass eaten }\end{array}$ & $\begin{array}{l}-0.041 \\
0.011\end{array}$ & 3560 & 0.8140 .932 & 0.9790 .891 & 17 & 44 & $\begin{array}{l}0.225 \\
0.328\end{array}$ \\
\hline & Feed duration & -0.258 & 60 & 0.047 & 0.019 & 9 & & 0.023 \\
\hline & Ingestion rate & 0.312 & 60 & 0.015 & 0.009 & 3 & & 0.041 \\
\hline
\end{tabular}

Data were processed such that no animal was represented more than once within a $33.3 \%$ segment of the stadium, but animals could be represented in two or in all three 1/3 segments (see text). Within-stadium comparisons of the initial and final one-third segments of the stadia (i.e. $0-33.3 \%$ versus $66.7-100 \%$ ) were made both using an independent samples design (Mann-Whitney test) and, for those animals represented in both segments,

a matched pairs design (Wilcoxon test).

$\mathrm{h}$ in the fifth. The 3.7 times greater increase in mass in the fifth stadium than in the fourth is the result of a 2.5 -fold increase in the rate of mass increase (size/age slope of 9.5 versus $3.8 \mathrm{mg} / \mathrm{h}$ ) for a 1.5 fold greater period ( $37.8 \mathrm{~h}$ versus $56 \mathrm{~h}$ ). Mass increased 7.5 -fold over in the fourth stadium and 4.5 -fold in the

fifth.

\section{Discussion}

Our data show that an important factor accounting for the developmental increase in food intake in $H$. armigera is the rate of food consumption, which increased between the fourth and fifth stadium, and also within the fifth stadium in the run-up to pupation. The story is, however, not a simple one, since an increase in the time spent feeding rather than ingestion rate was associated with increased food intake over fourth and the early stages of the fifth stadia.

We begin by considering our estimates of consumption, and then turn to a detailed discussion of the observed changes in feeding rates, including possible mechanisms and their relation to the data on feeding time reported in Raubenheimer and Barton Browne (2000). We end with a brief consideration of the possible ecological significance of our findings.

\subsection{Estimates of consumption}

In deriving our estimates of consumption by combining rates of ingestion measured in the present study with measures of time spent feeding from the previous study (Raubenheimer and Barton Browne, 2000), we have made two assumptions. The first is that the batches of larvae providing the two data sets had similar ontogenetic trajectories. We made every attempt to ensure this by carefully standardizing experimental conditions, including the same observers in the same constant temperature room using larvae from the same colony (only one or two generations removed) feeding on the same diet. There are strong indications that we were successful, because the fresh mass trajectories seen in the two studies were very similar and the duration of the fourth stadium (the only stadium for which there was this datum in the present study) seen in the two studies were almost identical. 
Our second assumption is that the rates of ingestion recorded in the present study were typical of the whole meal. In the earlier study which provided the data for total time spent feeding, a meal was considered terminated only following a pause of $240 \mathrm{~s}$. We tried in preliminary experiments to measure ingestion rate over meals so-defined but found that this approach gave highly variable results, because once the initial concerted feed had been broken by a pause the larvae often acted indecisively making it difficult to identify periods of actual ingestion. We therefore decided to measure ingestion rates only over the first uninterrupted period of feeding, and assume that they were representative of the meal. Although there must be some doubts as to the strict validity of this assumption, we believe the any consequential inaccuracies are minimal. For one, in our experimental setup there are relatively few breaks within meals, so the feeding bouts used to measure ingestion rates were a substantial proportion of the total period of ingestion in meals seen in our earlier study (ranging from median value of $40 \%$ in segment 3 of the fourth stadium to $80 \%$ in segment 2 of the fifth stadium). Therefore, even if there was a systematic change in rate of ingestion within a meal [e.g., a slowing over the course of a meal (Moorhouse et al., 1976)], this would not greatly affect the relativity of the estimates, and thus the patterns of

L. Barton Browne, D. Raubenheimer / Journal of Insect Physiology 49 (2003) 63-71

ontogenetic change. We are thus confident that patterns in food intake we derived across the fourth and fifth stadia are representative.

Our estimates showed that food consumption increased from the fourth to the fifth stadium by a factor of 3.5. Within the fourth stadium, there was no difference in estimated total consumption between the first two segments, but a ca 1.4-fold increase was observed from the second to the third segments. In the fifth stadium, intake increased progressively from the first to the third segments.

\subsection{Mechanisms of developmental changes in intake}

The observed 3.5-fold increase in intake in the fifth than the fourth stadium could have been achieved by one or a combination of the following: extending the feeding period of the stadium, increasing the amount of time spent feeding within the stadium, and increasing the rate at which food is ingested. When the whole stadium is considered, the observed difference is largely accounted for by the rate of ingestion being three times greater in the fifth than in the fourth stadium. The total time spent feeding in the fifth stadium was only ca 1.2 times that in the fourth. The 1.5 times longer period from ecdysis to the cessation of feeding in the fifth stadium was largely offset by a $21 \%$ reduction in the proportion of time spent feeding (Raubenheimer and Barton Browne, 2000).

The pattern seen over the whole stadium is reflected to varying degrees when comparisons are made between the corresponding segments of the two stadia. The most extreme case is seen in the segment 3 , where estimated consumption in the fifth stadium was ca 3.5 times that in the fourth stadium. This difference was more than fully accounted for by a 3.9 times greater rate of ingestion. In this instance, the total time spent feeding in this segment of the fifth stadium was only $89 \%$ of that in that of the fourth. The effect of the 1.5-fold greater duration of the fifth stadium segment was more than on offset by a $40 \%$ reduction in proportion of time spent feeding as compared with the same segment of the fourth stadium.

Unlike between-stadium comparisons, changes within stadia could have involved feeding rate and/or feeding time, but not alterations to the time available for feeding, since our comparisons were made between equal (33.3\%) time bins. In the fourth stadium estimated total consumption was fairly similar in the first two segments. This similarity resulted from there being similar rates of ingestion and similar times spent feeding in the two segments as opposed to differences of opposite sign in the two component variables. The ca 1.4 times greater estimated consumption in segment 3 than in segments 1 and 2 is fully accounted for by a ca 1.4-fold increase in the time spent feeding with rate of ingestion remaining similar across the three segments.

In the fifth stadium, estimated consumption increased progressively from segment to segment, but the basis for the increase between segments 2 and 3 was different from that for the increase between 
segments 1 and 2. The 1.3-fold greater estimated consumption in segment 2 than in segment 1 was the result of a 1.3-fold increase in time spent feeding, with rate of ingestion not differing significantly between the segments. On the other hand, the $c a 1$.3-fold increase between segment 2 and segment 3 occurred despite an ca $22 \%$ reduction in time spent feeding which was more than offset by a 1.6-fold increase in rate of ingestion.

\subsection{Mechanisms of altered time feeding and rates of ingestion}

A major component of the increase in food intake by

$H$. armigera with development was increased rate of ingestion, but increased feeding time was also observed between the second and third segments of the fourth stadium, and the first and second segments of the fifth.

Previously, we have determined that the mechanism underlying the increase in time spent feeding in both of these periods is a decrease in the duration of inter meal intervals (i.e. an increase in the number of meals taken per unit time) (Raubenheimer and Barton Browne, 2000).

Although we made no attempt to measure the fine-scaled behavioural events underlying the changes we observed in ingestion rate, they could involve an increase in the rate of biting and/or an increase in bite size. Because the head capsule of fifth instar larvae is substantially larger than that of fourth instar larvae (twofold difference in linear dimensions, unpublished observation), it is almost certain that bite size is larger in fifth stadium. Thus, if bite frequency were similar in these two stadia, as it is for $M$. sexta (Reynolds et al., 1986), increased bite size would explain the greater rate of ingestion in fifth instar larva. In contrast, the increased rate of ingestion observed within the fifth stadium clearly cannot be attributed to a change in the size of the head capsule. It could, nonetheless, be due to an increase in bite size which is somehow achieved despite the fixed size of the head capsule. One possibility is that larger bites are achieved due to an increase in the size of the mandibular muscles, which are known in caterpillars to change markedly with development (Bernays, 1986). The question would remain, however, as to why there was no similar increase in ingestion rate over the fourth stadium, where presumably the mandibular muscles also increased in size. Indeed, there was a greater proportionate increase in fresh mass over the feeding period of fourth stadium than over the fifth (ca 7.5-fold versus 4.4-fold), and the fresh mass trajectory is linear in both stadia.

Alternatively, increased feeding rates with develop

L. Barton Browne, D. Raubenheimer / Journal of Insect Physiology 49 (2003) 63-71

ment could have been due to an increase in bite frequency. As far as we are aware the only data relating bite frequency to stage of development within a stadium in a caterpillar are those obtained with fifth instar $M$. sexta by Reynolds et al. (1986) and Bowdan (1988), which are somewhat conflicting. Reynolds et al. reported that there was no significant variation over the course of the stadium in rate of ingestion or bite frequency when larvae were feeding either on an artificial diet or foliage, and concluded that bite size must be constant throughout. In contrast, Bowdan (1988) reported that bite frequency was positively correlated with insect mass in larvae feeding on foliage, and from this it seems plausible that the same might have been true in our study. There is, however, clearly need for a study of bite frequency and bite size (amount consumed over a feed divided by the number of bites) over the course of the two stadia.

\subsection{Might changes in ingestion rate be adaptive?}

An interesting feature of our results is the indication that the larvae can vary the amount of food consumed over a defined developmental period by varying the rate of ingestion, without there being, necessarily, a commensurate variation in time spent feeding (Fig. 1). Might these changes in the rate of ingestion be adaptive? We consider this possibility with particular reference to our finding that the rate of ingestion increased between segments 2 and 3 of the fifth stadium to such an extent that larvae 
consumed more food in segment 3 than in segment 2 despite a reduction in time spent feeding.

Predators and parasitoids are a major source of selection on caterpillars, with estimates for losses due to parasitoids alone ranging from 30\% to 95\% (Salazar and Whitman, 2001). It is, furthermore, almost certainly the case that caterpillars are particularly at risk when feeding. Bernays (1997) for example found that larvae of the two species of caterpillars suffered much higher rates of predation while feeding than while not feeding. This was demonstrated for early instars of Uresiphita reversalis being preyed upon by anthocorid bugs and third instar

M. sexta being preyed by a vespid wasp. Similarly, Gotthard (2000) showed that larvae of the wood butterfly (Pararge aegerua) that were manipulated using photoperiod to grow fast suffered higher predation than slower-growing controls, possibly because of the increased foraging activity required to sustain a higher growth rate. These results suggest that a benefit of rapid ingestion might be to enable the insect to satisfy its nutritional needs while minimizing the time spent feeding and, thus, reducing the risk of attack by natural enemies.

Predation might thus be one selective factor underlying the increase in feeding rate rather than feeding time that we observed in fifth instar larvae. A problem with this hypothesis, however, is that it begs the question of why it was the time spent feeding, rather than the rate of ingestion, that increased towards the end of the fourth stadium and again from the first to the second segment of the fifth. A functional explanation that took account of this would need to posit stage-specific cost-benefit relationships, and/or constraints on the rate of ingestion in H. armigera.

One possible constraint that prevented the smaller larvae from increasing the rate of ingestion is limitations on the strength of the mandibles and associated musculature. We consider this possibility unlikely, however, partly because the artificial diets we used were softer than most natural plant tissues, and so would not likely pose appreciable demands on the feeding apparatus. Also, Bernays (1986) demonstrated that the head and chewing musculature of the caterpillar Pseudaletia unipunctata was half the size when raised on soft artificial foods compared with hard grass even though body size was similar, showing that caterpillars have the developmental capacity to adapt their feeding apparatus to the nature of their foods. Since the caterpillars in our study had been reared on artificial foods from hatching (and for several generations), it would seem most likely that if maximizing feed rate was important the necessary adaptation would have taken place by the end of the penultimate larval stadium.

Rather than, or in addition to, constraints it might be that there are stage-specific selection pressures that explain the data. For example, caterpillars are known to face different sets of natural enemies as they grow (Salazar and Whitman, 2001), and the behaviour of different aged H. armigera might be adapted to the characteristics of their predominant enemies. There is good evidence that larger caterpillars are more vulnerable to predation by birds, while smaller size classes are more frequently taken by arthropod predators and parasitoids (reviewed by Montllor and Bernays, 1993; see also Cogni et al., 2002). Because birds are primarily visual foragers (Heinrich, 1993), there would be particularly strong incentive for the larger, late fifth instar, caterpillars to minimize the time during which they are feeding and so exposed to being seen by birds. Arthropod predators and parasitoids, by contrast, tend to use odour to locate their hosts (Salazar and Whitman, 2001). Fast feeding would result in high rate of release of volatiles from leaves, so creating a stronger odour plume, and is likely also messier than slower feeding. Slow feeding might have the additional benefit of increased digestive efficiency, if it results in the swallowing of more thoroughly chewed food (Slansky and Scriber, 1985; Slansky, 1993). Small larvae might thus on balance benefit from feeding more slowly compared with larger caterpillars.

While these suggestions remain speculative, they do serve to illustrate how data on the microstructure of feeding in insects can point the way towards interesting studies on the ecology of plant-insect interactions.

L. Barton Browne, D. Raubenheimer / Journal of Insect Physiology 49 (2003) 63-71

Further work which integrates the microstructure of caterpillar feeding with their ecology would likely yield important insights into the evolution of plant-insect interactions. 


\section{Acknowledgements}

The authors thank Dr R.E. Teakle and colleagues for providing larvae and experimental food and for useful discussions, and Drs Don Sands and Max Whitten for providing experimental facilities. They are also grateful to Professors Steve Simpson and Stuart Reynolds, Drs Marie-Louise Johnson and John Stanley and an anonymous referee for their comments on previous versions of the manuscript. Travel funds were provided to D.R. by grants from CSIRO, the Centre for Tropical Pest Management and the Royal Society. Behaviour recording equipment was purchased on a Nuffield Foundation

grant

to

D.R.

\section{References}

Barton Browne, L., 1995. Ontogenetic changes in feeding behavior. In: Chapman, R.F., de Boer, G. (Eds.), Regulatory Mechanisms in Insect Feeding. Chapman \& Hall, New York, pp. 307-342.

Bernays, E.A., 1986. Diet-induced head allometry among foliage-chewing insects and its importance for graminivores. Science 231, 495-497.

Bernays, E.A., 1991. Evolution of insect morphology in relation to plants. Philosophical Transactions of the Royal Society of London Series B, Biological Sciences 333, 257-264.

Bernays, E.A., 1997. Feeding by lepidopteran larvae is dangerous. Ecological Entomology 22, 121-123.

Bowdan, E., 1988. Microstructure of feeding by tobacco hornworm, Manduca sexta. Entomologia Experimentalis et Applicata 47, $127-136$.

Cogni, R., Freitas, A.L.V., Amaral, B.F., 2002. Influence of prey size on predation success by Zelus longipes L. (Het., Reduviidae). Journal of Applied Entomology 126, 74-78.

Gotthard, K., 2000. Increased risk of predation as a cost of high growth rate: an experimental test in the speckled wood butterfly, Pararge aegeria. Journal of Animal Ecology 69, 896-902.

Heinrich, B., 1993. How avian predators constrain caterpillar foraging. In: Stamp, N.E., Casey, T.M. (Eds.), Caterpillars: Ecological and Evolutionary Constraints on Foraging. Chapman \& Hall, New York, pp. 224-247.

Montllor, C.B., Bernays, E., 1993. Invertebrate predators and caterpillar foraging. In: Stamp, N.E., Casey, T.M. (Eds.), Caterpillars: Ecological and Evolutionary Constraints on Foraging. Chapman \& Hall, New York, pp. 170-202.

Moorhouse, J.E., Barton Browne, L., van Gerwen, A.C.M., 1976. Factors affecting the rate of ingestion of liquids by the locust, Chortoicetes terminifera. Journal of Insect Physiology 22, 259-263.

Phillipson, J., 1981. Bioenergetic options and phylogeny (Chapter 2). In: Townsend, C.R., Calow, P. (Eds.), Physiological Ecology: An Evolutionary Approach to Resource Use. Blackwell Scientific, Oxford, pp. 20-45.

Raubenheimer, D., Barton Browne, L., 2000. Developmental changes on the patterns of feeding in fourth and fifth instar Helicoverpa armigera caterpillars. Physiological Entomology 25, 390-399.

Reynolds, S.E., Nottingham, S.F., Stephens, A.E., 1985. Food and water economy and its relation to growth in fifth-instar larvae of the tobacco hornworm, Manduca sexta. Journal of Insect Physiology 31, 119-127.

Reynolds, S.E., Yeomans, M.R., Timmins, W.A., 1986. The feeding behaviour of caterpillars (Manduca sexta) on tobacco and on artificial diet. Physiological Entomology 11, 39-51.

Salazar, B.A., Whitman, D.W., 2001. Defensive tactics of caterpillars against predators and parasitoids. In: Ananthakrishnan, T.N. (Ed.), Insects and Plant Defence Dynamics. Science Publishers, Plymouth, pp. 161-207.

Schroeder, L.A., 1981. Consumer growth efficiencies: their limits and relationships to ecological energetics. Journal of Theoretical Biology 93, 805-828.

Slansky, F. Jr, 1993. Nutritional ecology: the fundamental quest for nutrients. In: Stamp, N.E., Casey, T.M. (Eds.), Caterpillars: Ecological and Evolutionary Constraints on Foraging. Chapman \& Hall, New York, pp. 29-91.

Slansky, F., Scriber, J.M., 1985. Food consumption and utilization. In: Kerkut, G.A., Gilbert, L.I. (Eds.), Comprehensive Insect Physiology, Biochemistry, and Pharmacology, vol. 4. Pergamon, Oxford, pp. 87-163.

Teakle, R.E., Jensen, J.M., 1985. Rearing Heliothis armigera. In: Singh, B.D., Moore, R.F. (Eds.), Handbook of Insect Rearing, vol.
2.
Elsevier,
New
York,
pp.
313-322. 